Punjab University Journal of Mathematics (2021),53(5),329-336

https://doi.org/10.52280/pujm.2021.530503

\title{
Integrity of Wheel Related Graphs
}

\author{
${ }^{*}$ B. Basavanagoud and ${ }^{1}$ Shruti Policepatil \\ ${ }^{*}, 1$ Department of Mathematics, \\ Karnatak University, Dharwad - 580 003, Karnataka, India. \\ Email: *b.basavanagoud@gmail.com; \\ ${ }^{1}$ shrutipatil300@gmail.com
}

Received: 12 January, 2021 / Accepted: 06 April, 2021 / Published online: 26 May, 2021

\begin{abstract}
If a network modeled by a graph, then there are various graph theoretical parameters used to express the vulnerability of communication networks. One of them is the concept of integrity. In this paper, we determine exact values for the integrity of wheel related graphs.
\end{abstract}

\section{AMS (MOS) Subject Classification Codes: 05C40; 05C69; 90C35}

Key Words: Integrity, wheel related graphs, communication network.

\section{INTRODUCTION}

In this paper, we consider simple, finite, undirected graphs. Let $G$ be a graph with a vertex set $V(G)$ and an edge set $E(G)$ such that $|V(G)|=n$ and $|E(G)|=m$. we refer to Harary [13] for notations and terminologies not defined here.

The stability of a communication network composed of processing nodes(vertices) and communication links(edges) are of primary importance to network designers. As the network begins losing links or nodes, eventually there will be a decrease in its effectiveness. Thus, communication networks constructed as stable as possible, not only with respect to the earliest damage, but also with respect to the possible reformation of the network. Integrity is the best parameter to measure the stability of network.

Barefoot et al. in [6] introduced the concept of integrity. The integrity of a graph $G$ is defined as

$$
I(G)=\min _{S \subset V(G)}\{|S|+m(G-S)\},
$$

where $m(G-S)$ denotes the order of the largest component of $G-S$. In [6], the authors have compared integrity, connectivity, toughness and binding number for several classes of graphs. In 1987, Barefoot et al. [7] have investigated the integrity of trees and powers of cycles. In 1988, Goddard et al. [11] have obtained integrity of the join, union, product 
and composition of two graphs. The authors in $[2,14]$ have studied the integrity of cubic graphs. Inspired by this, we obtain integrity of wheel related graphs. For more details on integrity of a graph refer to $[3,4,5,8,10,12]$.

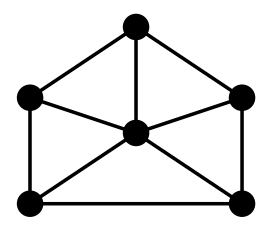

Wheel Graph, $W_{5}$

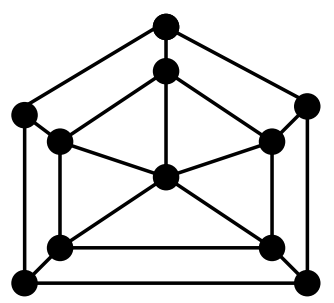

Closed Helm Graph, $\mathrm{CH}_{5}$

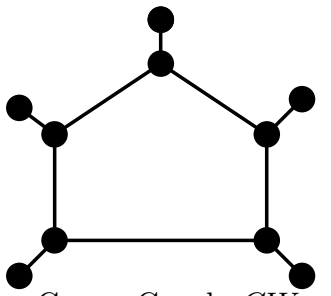

Crown Graph, $C W_{5}$

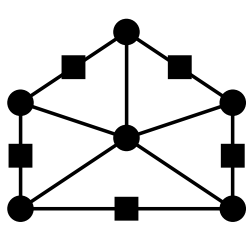

Gear Graph, $G_{5}$

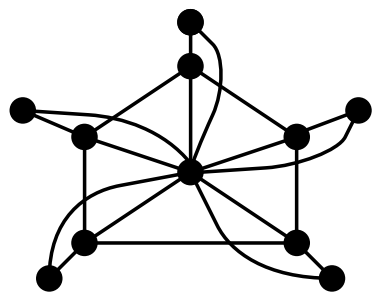

Flower Graph, $F l_{5}$

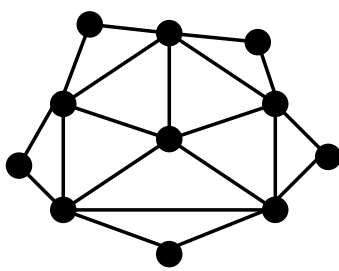

Sunflower Graph, $S F_{5}$
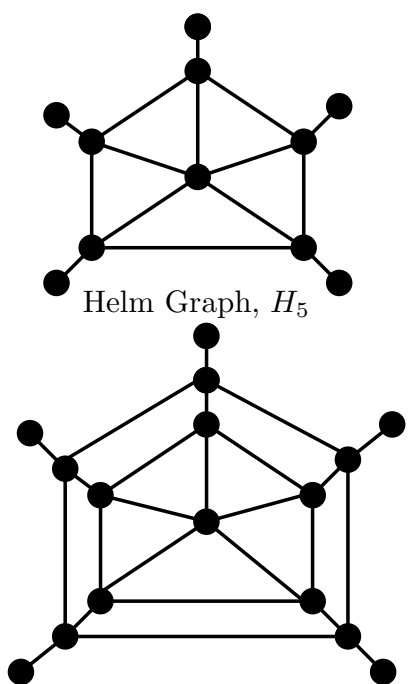

Web Graph, $W(2,5)$

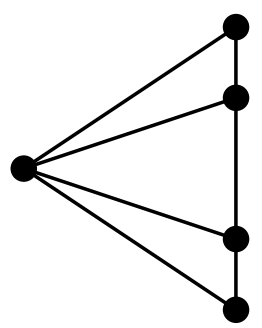

Fan Graph, $F_{5}$

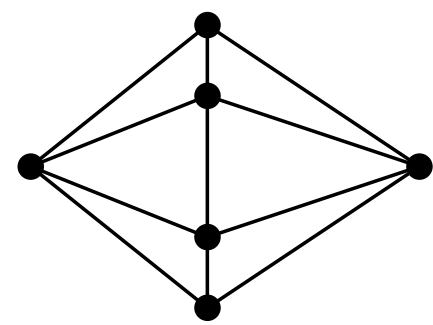

Double Fan Graph, $D F_{5}$

FIGURE 1. Some wheel related graphs 


\section{PRELIMINARIES}

We list some of the basic results.

Theorem 2.1. [5] The integrity

(i) complete graph $K_{n}, I\left(K_{n}\right)=n$,

(ii) null graph $\overline{K_{n}}, I\left(\overline{K_{n}}\right)=1$,

(iii) star $K_{1, n}, I\left(K_{1, n}\right)=2$,

(iv) path $P_{n}, I\left(P_{n}\right)=\lceil 2 \sqrt{n+1}\rceil-2$,

(v) cycle $C_{n}, I\left(C_{n}\right)=\lceil 2 \sqrt{n}\rceil-1$,

(vi) complete bipartite graph $K_{a, b}, I\left(K_{a, b}\right)=1+\min \{a, b\}$,

(vii) wheel $W_{n}, I\left(W_{n}\right)=\lceil 2 \sqrt{n-1}\rceil$.

Theorem 2.2. [5] For any graphs $G$ and $H$,

$$
I(G+H)=\min \{I(G)+|H|, I(H)+|G|\} .
$$

\section{INTEGRITY OF WHEEL RELATED GRAPHS}

In this section, we obtain integrity of wheel related graphs.

Definition 3.1. [9] The graph $W_{n}=K_{1}+C_{n}$ is called a wheel graph. In wheel graph, the vertex c of degree $n$ is called the central vertex while the vertices on the cycle $C_{n}$ are called rim vertices.

Definition 3.2. [9] The gear graph $G_{n}$ is obtained from a wheel graph by inserting a vertex between each pair of adjacent vertices of the outer cycle.

Theorem 3.3. For a gear graph $G_{n}$ of order $2 n+1$,

$$
I\left(G_{n}\right)= \begin{cases}n+1, & \text { if } 3 \leq n \leq 7 \\ \lceil 2 \sqrt{2 n}\rceil, & \text { otherwise }\end{cases}
$$

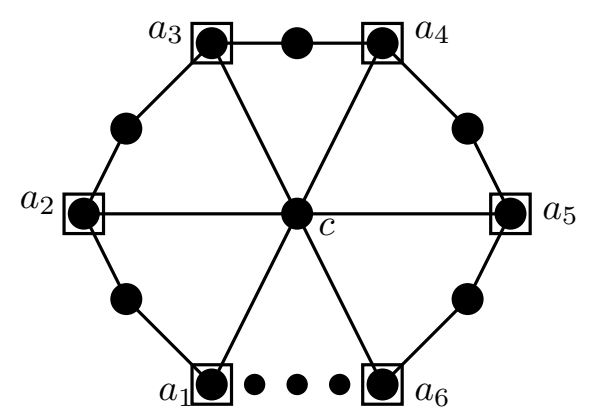

(a)

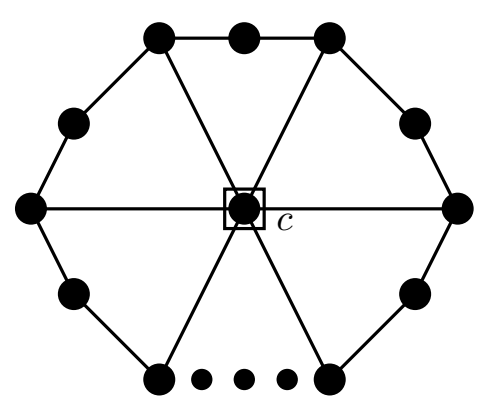

(b)

FIGURE 2. Choosing $\mathrm{S}$ in gear graph.

Proof. Let $S$ be the collection of some rim vertices and central vertex $c$. Let $S \subset V\left(G_{n}\right)$. To choose $S$, we have two cases. 
Case 1. If $3 \leq n \leq 7$, then choose set $S=\left\{a_{i}: i=1,2, \ldots, n\right\}$ in such a way that it is containing all vertices adjacent to central vertex of $G_{n}$ (as shown in Figure 2(a)). It is clear to write $|S|=n$. $G_{n}-S$ results in a graph with $n+1$ components of order 1 . Hence, $m\left(G_{n}-S\right)=1$. $|S|+m\left(G_{n}-S\right)$ is minimum for the above defined set $S$. Thus, $I\left(G_{n}\right)=n+1$.

Case 2. Suppose $n \geq 8$. Then choose $S=\{c\}$, where $c$ is central vertex of gear graph (as shown in Figure 2(b)). Then, $G_{n}-S$ will be the cycle of order $2 n$.

Therefore, $I\left(G_{n}\right)=|S|+I\left(C_{2 n}\right)$. By Theorem 2.1(v), we get the required result.

Definition 3.4. [9] The helm $H_{n}$ is a graph obtained from a wheel $W_{n}$ with central vertex $c$, by attaching a pendant edge to each rim vertex of $W_{n}$.

Theorem 3.5. For a helm graph $H_{n}$ of order $2 n+1$,

$$
I\left(H_{n}\right)= \begin{cases}\left\lceil\frac{n}{2}\right\rceil+3, & \text { if } 3 \leq n \leq 10 \\ \left\lceil\frac{n}{3}\right\rceil+5, & \text { otherwise }\end{cases}
$$

Proof. Let $S$ be the collection of some rim vertices and central vertex $c$. Let $S \subset V\left(H_{n}\right)$. The set $S$ can be chosen in two ways.

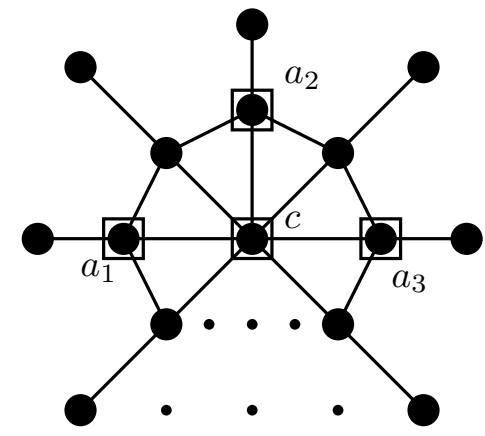

(a)

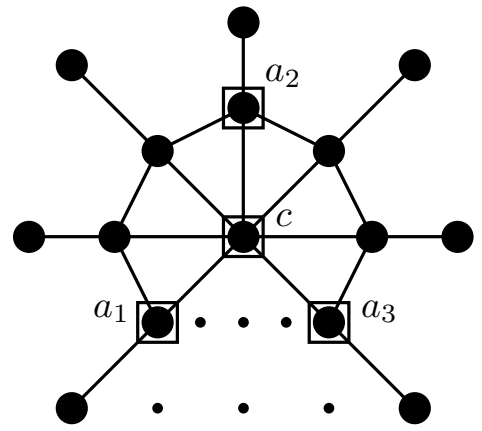

(b)

FIgURE 3. Choosing $\mathrm{S}$ in helm graph.

case 1. Suppose $3 \leq n \leq 10$. Then $S=\left\{a_{i}: i=1,2, \ldots,\left\lceil\frac{n}{2}\right\rceil\right\} \cup\{c\}$ (as shown in Figure 3 (a)) such that $d\left(a_{1}, a_{\left\lceil\frac{n}{2}\right\rceil}\right)=1$ or 2 and $d\left(a_{i}, a_{j}\right)=2$, where $i \neq j, j=1,2, \ldots,\left\lceil\frac{n}{2}\right\rceil$. Clearly, $|S|=\left\lceil\frac{n}{2}\right\rceil+1$. $H_{n}-S$ is a graph with components $K_{1}$ and $K_{2}$.

Hence, $m\left(H_{n}-S\right)=2$. The set $S$ gives least value of $|S|+m\left(H_{n}-S\right)$ which gives the value of integrity. Thus, $I\left(H_{n}\right)=\left\lceil\frac{n}{2}\right\rceil+3$.

Case 2. If $n \geq 11$, then $S=\left\{a_{i}: i=1,2, \ldots,\left\lceil\frac{n}{3}\right\rceil\right\} \cup\{c\}$ (as shown in Figure 3(b)) such that $d\left(a_{1}, a_{\left\lceil\frac{n}{3}\right\rceil}\right)=1$ or 2 or 3 and $d\left(a_{i}, a_{j}\right)=3$, where $i \neq j, j=1,2, \ldots,\left\lceil\frac{n}{3}\right\rceil$. Clearly, $|S|=\left\lceil\frac{n}{3}\right\rceil+1$. $H_{n}-S$ results in a graph with components of order 1,2 and 4. Hence, $m\left(H_{n}-S\right)=4 .|S|+m\left(H_{n}-S\right)$ is least for the above defined set $S$. Thus, $I\left(H_{n}\right)=\left\lceil\frac{n}{3}\right\rceil+5$. 
Definition 3.6. [9] The flower graph $\mathrm{Fl}_{n}$ is the graph obtained from a helm $H_{n}$ by joining each pendant vertex to the central vertex $c$ of the helm.

Theorem 3.7. For a flower graph $F l_{n}$ of order $2 n+1(n \geq 4)$,

$$
I\left(F l_{n}\right)= \begin{cases}\left\lceil\frac{n}{2}\right\rceil+3, & \text { if } 4 \leq n \leq 10, \\ \left\lceil\frac{n}{3}\right\rceil+5, & \text { otherwise. }\end{cases}
$$

Proof. The proof is similar to that of Theorem 3.5.

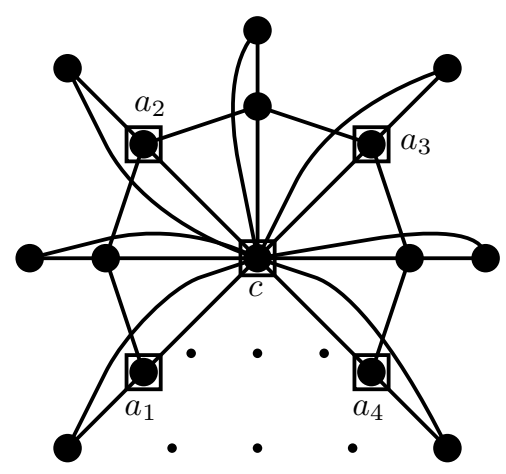

(a)

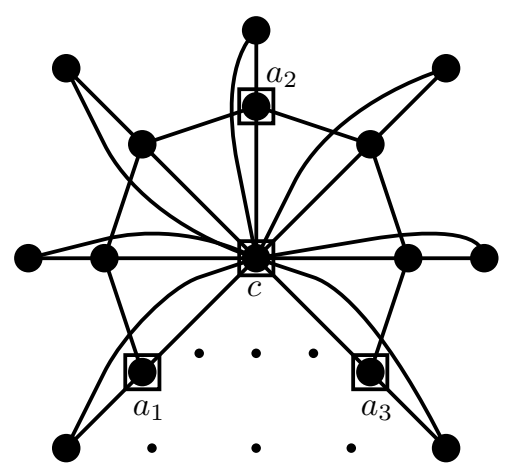

(b)

FIGURE 4. Choosing $\mathrm{S}$ in flower graph.

Remark 3.8. From the Theorems 3.5 and 3.7, we observe that the integrities of flower graph and helm graph are equal for $n \geq 4$.

Definition 3.9. [9] The crown (or sun) $C W_{n}$ is a corona of form $C_{n} \circ K_{1}$ where $n \geq 3$. That is crown is a helm without a central vertex.

Theorem 3.10. For a crown graph $C W_{n}$ order $2 n(n \geq 3)$,

$$
I\left(C W_{n}\right)= \begin{cases}\left\lceil\frac{n}{2}\right\rceil+2, & \text { if } 4 \leq n \leq 10 \\ \left\lceil\frac{n}{3}\right\rceil+4, & \text { otherwise }\end{cases}
$$

Proof. Let $S$ be the collection of some rim vertices and central vertex $c$. Let $S \subset V\left(C W_{n}\right)$. The set $S$ can be chosen in two ways.

case 1. If $3 \leq n \leq 10, S=\left\{a_{i}: i=1,2, \ldots,\left\lceil\frac{n}{2}\right\rceil\right\}$ (as shown in Figure 5(a)) such that $d\left(a_{1}, a_{\left\lceil\frac{n}{2}\right\rceil}\right)=1$ or 2 and $d\left(a_{i}, a_{j}\right)=2$, where $i \neq j, j=1,2, \ldots,\left\lceil\frac{n}{2}\right\rceil$. Clearly, $|S|=\left\lceil\frac{n}{2}\right\rceil . C W_{n}-S$ is a graph with components $K_{1}$ and $K_{2}$. Hence, $m\left(C W_{n}-S\right)=2$. The set $S$ gives least value of $|S|+m\left(C W_{n}-S\right)$ which gives the value of integrity. Thus, $I\left(C W_{n}\right)=\left\lceil\frac{n}{2}\right\rceil+2$.

Case 2. If $n \geq 11, S=\left\{a_{i}: i=1,2, \ldots,\left\lceil\frac{n}{3}\right\rceil\right\}$ (as shown in Figure 5(b)) such that $d\left(a_{1}, a_{\left\lceil\frac{n}{3}\right\rceil}\right)=1$ or 2 or 3 and $d\left(a_{i}, a_{j}\right)=3$, where $i \neq j, j=1,2, \ldots,\left\lceil\frac{n}{3}\right\rceil$. Clearly, $|S|=\left\lceil\frac{n}{3}\right\rceil . C W_{n}-S$ results in a graph with components of order 1,2 and 4 . Hence, 
$m\left(C W_{n}-S\right)=4 .|S|+m\left(C W_{n}-S\right)$ is least for the above defined set $S$. Thus, $I\left(C W_{n}\right)=\left\lceil\frac{n}{3}\right\rceil+4$.
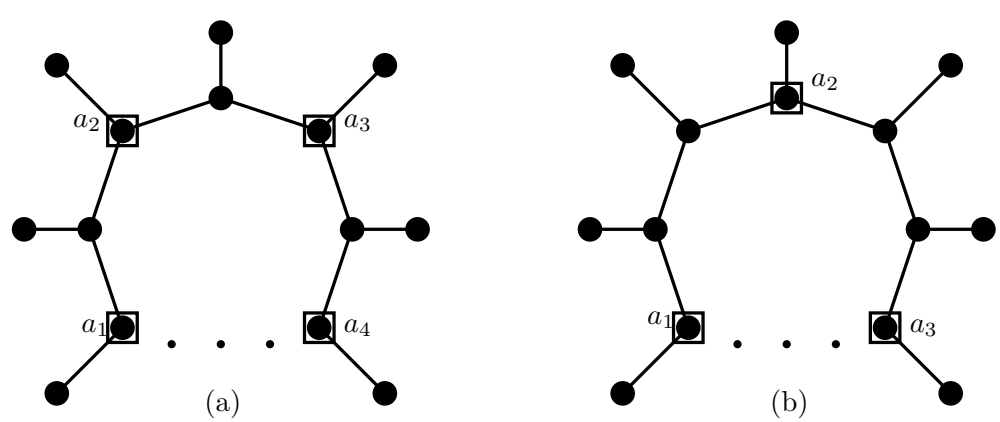

FiguRE 5. Choosing $\mathrm{S}$ in crown graph.

Corollary 3.11. For a crown graph $C W_{n}$ order $2 n(n \geq 3)$,

$$
I\left(C W_{n}\right)=I\left(H_{n}\right)-1 .
$$

Definition 3.12. [9] The closed helm $\mathrm{CH}_{n}$ is the graph with central vertex c, obtained from a helm by joining each pendant vertex to form a cycle.

Theorem 3.13. For a closed helm graph $\mathrm{CH}_{n}$ of order $2 n+1$,

$$
I\left(C H_{n}\right)= \begin{cases}2\left\lceil\frac{n}{2}\right\rceil+2, & \text { if } 3 \leq n \leq 10 \\ 2\left\lceil\frac{n}{4}\right\rceil+7, & \text { otherwise. }\end{cases}
$$

Proof. We have to choose $S_{1}$ from rim vertices of inner cycle and $S_{2}$ from rim vertices of outer cycle such that $\left|S_{1}\right|=\left|S_{2}\right|$. Let $c$ be a central vertex. The set $S$ can be chosen in two ways.

Case 1. If $3 \leq n \leq 10$, choose $S_{1}=\left\{a_{i}: i=1,2, \ldots,\left\lceil\frac{n}{2}\right\rceil\right\}$ (as shown in Figure 6(a)) such that $d\left(a_{1}, a_{\left\lceil\frac{n}{2}\right\rceil}\right)=1$ or 2 and $d\left(a_{i}, a_{j}\right)=2 . S_{2}=\left\{b_{i}: i=1,2, \ldots,\left\lceil\frac{n}{2}\right\rceil\right\}$ such that $d\left(b_{1}, b_{\left\lceil\frac{n}{2}\right\rceil}\right)=1$ or 2 and $d\left(b_{i}, b_{j}\right)=2$, where $i \neq j, j=1,2, \ldots,\left\lceil\frac{n}{2}\right\rceil . a_{1}$ must not be adjacent to $b_{1} . S=S_{1} \cup S_{2} \cup\{c\}$. So, $|S|=2\left\lceil\frac{n}{4}\right\rceil+1$. $C H_{n}-S$ results in a disconnected graph with components of order 1 . Hence, $m\left(C H_{n}-S\right)=1$. The set $S$ defined above gives minimum value of $|S|+m\left(C H_{n}-S\right)$ which gives the value of integrity. Thus, $I\left(\mathrm{CH}_{n}\right)=2\left\lceil\frac{n}{2}\right\rceil+2$.

Case 2. If $n \geq 11$, choose $S_{1}=\left\{a_{i}: i=1,2, \ldots,\left\lceil\frac{n}{4}\right\rceil\right\}$ (as shown in Figure 6(b)) such that $d\left(a_{1}, a_{\left\lceil\frac{n}{4}\right\rceil}\right)=1$ or 2 or 3 or 4 and $d\left(a_{i}, a_{j}\right)=4 . S_{2}=\left\{b_{i}: i=1,2, \ldots,\left\lceil\frac{n}{4}\right\rceil\right\}$ such that $d\left(b_{1}, b_{\left\lceil\frac{n}{4}\right\rceil}\right)=1$ or 2 or 3 or 4 and $d\left(b_{i}, b_{j}\right)=4$. where $i \neq j, j=1,2, \ldots,\left\lceil\frac{n}{4}\right\rceil . a_{1}$ must be adjacent to $b_{1} . S=S_{1} \cup S_{2} \cup\{c\}$. So, $|S|=2\left\lceil\frac{n}{4}\right\rceil+1$. $C H_{n}-S$ results in a disconnected graph with components of order 2 or 4 or 6 . Hence, $m\left(\mathrm{CH}_{n}-S\right)=6$. $|S|+m\left(\mathrm{CH}_{n}-S\right)$ is least for above set $S$, the value of integrity. Thus, $I\left(\mathrm{CH}_{n}\right)=2\left\lceil\frac{n}{4}\right\rceil+7$. 


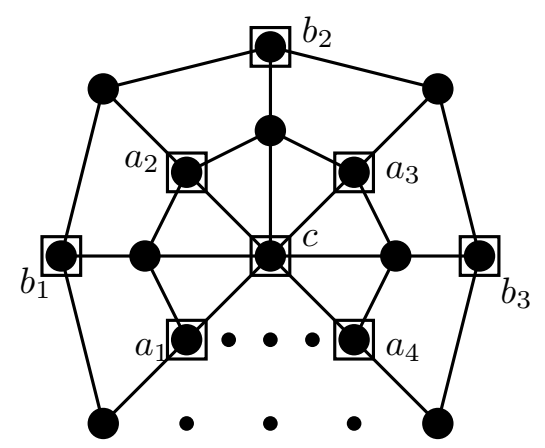

(a)

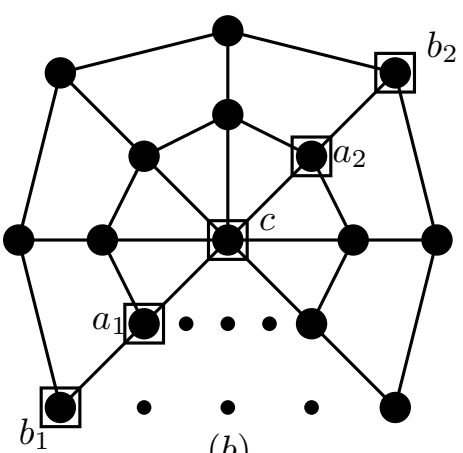

(b)

FIGURE 6. Choosing $\mathrm{S}$ in closed helm graph.

Definition 3.14. [9] A web graph is the graph obtained by joining a pendant edge to each vertex on the outer cycle of the closed helm. $W(t, n)$ is the generalized web with $t$ cycles each of order $n$.

Theorem 3.15. Let $W(t, n)$ be a web graph of order $3 n+1(n \geq 3)$. Then,

$$
I(W(t, n))=n+3 .
$$
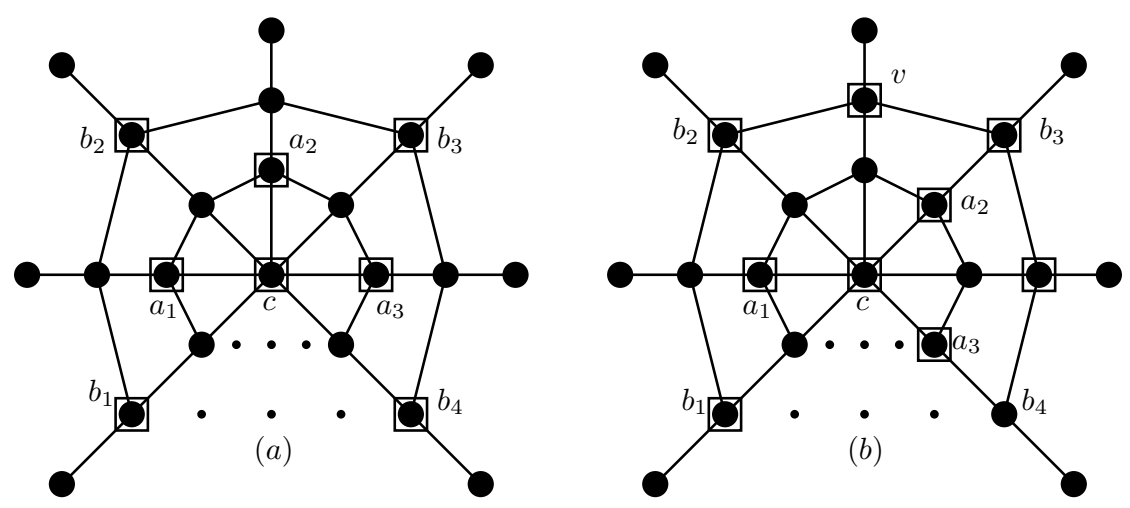

Figure 7. Choosing $\mathrm{S}$ in web graph.

Proof. Let $S_{1}=\left\{a_{i}: i=1,2, \ldots, \frac{n}{2}.\right\}$ be the maximal independent set of vertices of outer cycle and $S_{2}=\left\{b_{i}: i=1,2, \ldots, \frac{n}{2} \cdot\right\}$ be the maximal independent set of vertices of inner cycle, where $a_{i}$ is not adjacent to $b_{i}$. Let $c$ be a central vertex. We have two cases to choose the set $S$.

Case 1. If $\mathrm{n}$ is even, $S=S_{1} \cup S_{2} \cup\{c\}$ (as shown in Figure 7(a)). Clearly, $|S|=$ $2 \beta_{0}\left(C_{n}\right)+1$, where $\beta_{0}$ is point independence number. Thus, $|S|=n+1$.

Case 2. If $n$ is odd, choose vertex $v$ from an outer cycle (or inner cycle) of $W(t, n)$ such 
that $v$ is adjacent to vertices of $S_{1}\left(\right.$ or $S_{2}$ ). So, $S=S_{1} \cup S_{2} \cup\{v\} \cup\{c\}$ (as shown in Figure 7(b)). So, $|S|=2 \beta_{0}\left(C_{n}\right)+2$, where $\beta_{0}$ is point independence number. Therefore $|S|=n+1$.

From the above two cases $|S|=n+1 . W(t, n)-S$ results in a disconnected graph with components $K_{1}$ and $K_{2}$. Hence, $m(W(t, n)-S)=2$. The set $S$ defined above gives least value of $|S|+m(W(t, n)-S)$. Thus, $I(W(t, n))=n+3$.

Definition 3.16. [9] The sunflower graph $S F_{n}$ is a graph obtained from a wheel with central vertex $c, n$-cycle $v_{0}, v_{1}, \ldots, v_{n-1}$ and additional $n$ vertices $w_{0}, w_{1}, \ldots, w_{n-1}$ where $w_{i}$ is joined by edges to $v_{i}, v_{i+1}$ for $i=0,1, \ldots, n-1$ where $i+1$ is taken modulo $n$.

Theorem 3.17. Let $S F_{n}$ be a sunflower graph of order $2 n+1$. Then,

$$
I\left(S F_{n}\right)= \begin{cases}n+1, & \text { if } 3 \leq n \leq 5, \\ \left\lceil\frac{n}{2}\right\rceil+4, & \text { if } 6 \leq n \leq 8, \\ \left\lceil\frac{n}{3}\right\rceil+6, & \text { otherwise. }\end{cases}
$$

Proof. Let $S \subset V\left(S F_{n}\right)$. The set $S$ can be chosen in two ways.

Case 1. If $3 \leq n \leq 5$, then choose set $S$ containing all vertices adjacent to central vertex of $S F_{n}$. It is clear to write $|S|=n . S F_{n}-S$ results in a graph with $n+1$ components of order 1. Hence, $m\left(S F_{n}-S\right)=1$. The set $S$ gives minimum value of $|S|+m\left(S F_{n}-S\right)$. Thus, $I\left(S F_{n}\right)=n+1$.

Case 2. Suppose $6 \leq n \leq 8$. Then choose $S_{1}=\left\{a_{i}: i=1,2, \ldots,\left\lceil\frac{n}{2}\right\rceil\right\}$ (as shown in Figure 8(a)) such that $d\left(a_{1}, a_{\left\lceil\frac{n}{2}\right\rceil}\right)=1$ or 2 and $d\left(a_{i}, a_{j}\right)=2$, where $i \neq j, j=$ $1,2, \ldots,\left\lceil\frac{n}{2}\right\rceil$. Let $S=S_{1} \cup\{c\}$. So, $|S|=\left\lceil\frac{n}{2}\right\rceil+1 . S F_{n}-S$ results in a disconnected graph with components of order 1 or 3 . Hence, $m\left(S F_{n}-S\right)=3$. Therefore, $I\left(S F_{n}\right)=\left\lceil\frac{n}{2}\right\rceil+4$. Case 3. If $n \geq 9$, choose $S_{2}=\left\{b_{i}: i=1,2, \ldots,\left\lceil\frac{n}{3}\right\rceil\right\}$ (as shown in Figure 8(b)) such that $d\left(b_{1}, b_{\left\lceil\frac{n}{3}\right\rceil}\right)=2$ and $d\left(b_{i}, b_{j}\right)=2$, where $i \neq j, j=1,2, \ldots,\left\lceil\frac{n}{3}\right\rceil$. Let $S=S_{2} \cup\{c\}$. So, $|S|=\left\lceil\frac{n}{3}\right\rceil+1 . S F_{n}-S$ results in a disconnected graph with components of order 3 or 5 . Hence, $m\left(S F_{n}-S\right)=5$. The set $S$ gives minimum value of $|S|+m\left(S F_{n}-S\right)$. Thus, $I\left(S F_{n}\right)=\left\lceil\frac{n}{3}\right\rceil+6$.

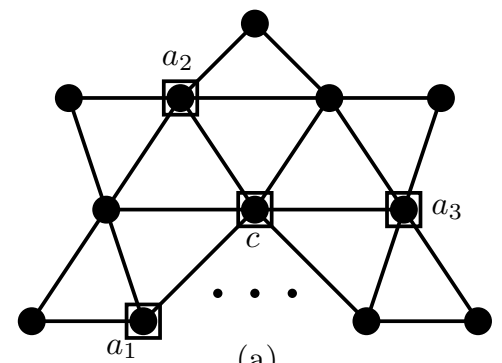

(a)

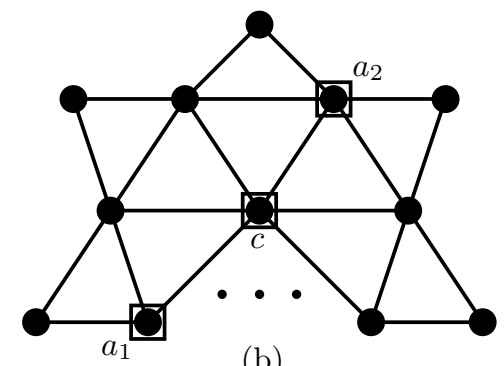

(b)

FIGURE 8. Choosing $\mathrm{S}$ in sunflower graph.

Definition 3.18. [9] The friendship graph $C_{3}^{(t)}$ is a collection of t-triangles with a common vertex. Friendship graph can also be obtained from a wheel $W_{2 n}$ with cycle $C_{2 n}$ by deleting alternate edges of the cycle. That is $f_{n}=K_{1}+n K_{2}$. 
Theorem 3.19. For a Friendship graph $C_{3}^{(t)}$ of order $2 t+1$,

$$
I\left(C_{3}^{(t)}\right)=3 \text {. }
$$

Proof. Choose $S=\{c\}$, where c is a central vertex. Clearly, $|S|=1$. Then, the graph $C_{3}^{(t)}-S \cong t K_{2}$. Hence, $m\left(C_{3}^{(t)}-S\right)=2$. The set $S$ defined above gives least value of $|S|+m\left(C_{3}^{(t)}-S\right)$, the value of integrity. Thus, $I\left(C_{3}^{(t)}\right)=3$.

Example 3.20. Consider a graph $C_{3}^{(4)}$. Let $S=\{c\} \subset V\left(C_{3}^{(4)}\right)$ (see Figure 9) such that $|S|=1$ and $m\left(C_{3}^{(4)}-S\right)=2$. Therefore, $I\left(C_{3}^{(4)}\right)=3$.

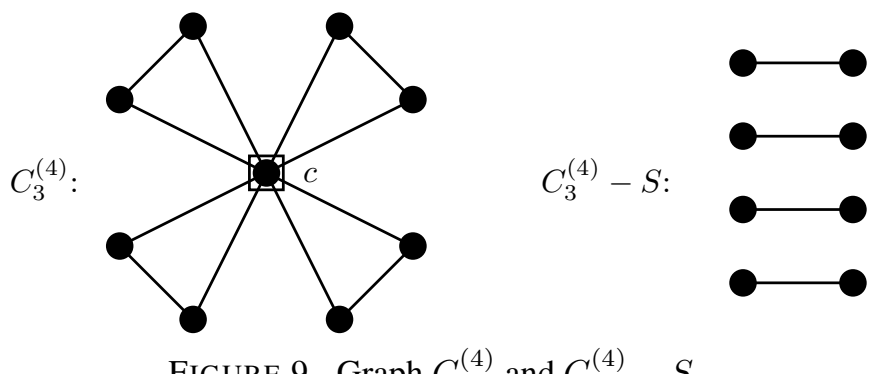

FiguRE 9. Graph $C_{3}^{(4)}$ and $C_{3}^{(4)}-S$.

Definition 3.21. [9] The fan graph $F_{n}(n \geq 3)$ is defined as the graph $K_{1}+P_{n-1}$, where $K_{1}$ is singleton graph and $P_{n-1}$ is the path on $n-1$ vertices.

Theorem 3.22. Let $F_{n}$ be a fan graph of order $n \geq 3$. Then

$$
I\left(F_{n}\right)=\lceil 2 \sqrt{n}\rceil-1 .
$$

Proof. The fan graph is join of $K_{1}$ and $P_{n-1}$. So, $I\left(F_{n}\right)=I\left(K_{1}+P_{n-1}\right)$. Thus, by Theorems 2.1(iv) and 2.2, we get the desired result.

Definition 3.23. [9] The double fan graph $d f_{n}(n \geq 3)$ is defined as the graph $2 K_{1}+P_{n-1}$.

Theorem 3.24. For a double fan $D F_{n}$ of order $n+1(n \geq 3)$,

$$
I\left(D F_{n}\right)=\lceil 2 \sqrt{n}\rceil .
$$

Proof. The double fan graph is join of $2 K_{1}$ and $P_{n-1}$. So, $I\left(D F_{n}\right)=I\left(2 K_{1}+P_{n-1}\right)$. From Theorems 2.1(iv) and 2.2, we get the desired result.

\section{CONCLUSiON}

In this paper, we have concentrated on integrity, a measure of network vulnerability. We have computed the integrity of wheel related graphs. Also, we have established the relationship between some wheel related graphs. Wheel related graphs are taken to model the network system and the integrity values of them reveal that how network can be made more stable than earlier. These results can help the network designers to choose a suitable topology for the network. This study can be very useful in the investigation of complex network robustness. 


\section{REFERENCES}

[1] M. Atici and A. Kirlangiç, Counter examples to the theorems of integrity of prism and ladders, J. Combin. Math. Combin. Comp., 34 (2000) 119-127.

[2] M. Atici, R. Crawford and C. Ernst, New upper bounds for the integrity of cubic graphs, Int. J. Comput. Math., 81, No. 11 (2004) 1341-1348.

[3] A. Aytaç and S. Çelik, Vulnerability: Integrity of a middle graph, Selçuk J. Appl. Math., 9, No. 1 (2008) 49-60.

[4] K. S. Bagga, L. W. Beineke, M. J. Lipman and R. E. Pippert, The integrity of the prism (Preliminary Report), Abstracts Amer. Math. Soc., 10 (1989) pp. 12.

[5] K. S. Bagga, L. W. Beineke, W. D. Goddard, M. J. Lipman and R.E. Pippert, A survey of integrity, Discrete Appl. Math., 37 (1992) 13-28.

[6] C. A. Barefoot, R. Entringer and H C. Swart, Vulnerability in graphs - A comparitive survey, J. Combin. Math. Combin. Comput., 1 (1987) 13-21.

[7] C. A. Barefoot, R. Entringer and H. C. Swart, Integrity of trees and powers of cycles, Congr. Numer., 58 (1987) 103-114.

[8] P. Dündar and A. Aytaç, Integrity of total graphs via certain parameters, Math. Notes, 76, No. 5 (2004) 665-672.

[9] J. A. Gallian: A dynamic survey of graph labeling, Electron. J. Combin. \#DS6, 2018.

[10] W. Goddard, On the vulnerability of graphs, Ph.D. Thesis, University of Natal, Durban, S.A., (1989).

[11] W. D. Goddard and H. C. Swart, On the integrity of combinations of graphs, J. Combin. Math. Combin. Comp., 4 (1988) 3-18.

[12] W. D. Goddard and H. C. Swart, Integrity in graphs: Bounds and basics, J. Combin. Math. Combin. Comp., 7 (1990) 139-151.

[13] F. Harary: Graph Theory, Addison-Wesley, Reading, 1969.

[14] A. Vince, The integrity of a cubic graph, Discrete Appl. Math., 140 (2004) 223-239. 